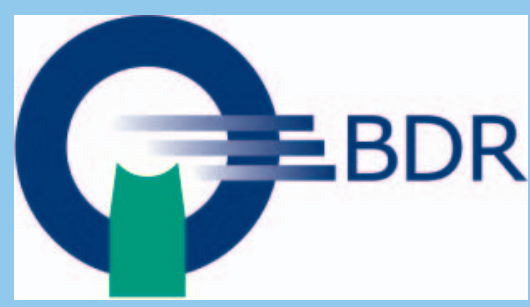

\title{
Interessante Buchempfehlungen für RadiologInnen - Lesen in der Krise
}

Vielleicht haben Sie jetzt etwas Zeit zur Fortbildung im „Homeoffice“? BDR-Mitglieder rezensieren .... Regelmäßig veröffentlichen wir im RADIOLOGEN interessante Besprechungen von radiologischen und gesundheitspolitischen Fachbüchern. Einige davon, aus dem Thieme-Verlag, finden Sie in dieser Ausgabe der RöFo.

Weitere Rezensionen finden Sie auf unserer Webseite www.radiologenverband.de.

\section{Neuromuscular Spine Deformity}

\section{Neuromuscular Spine Deformity}

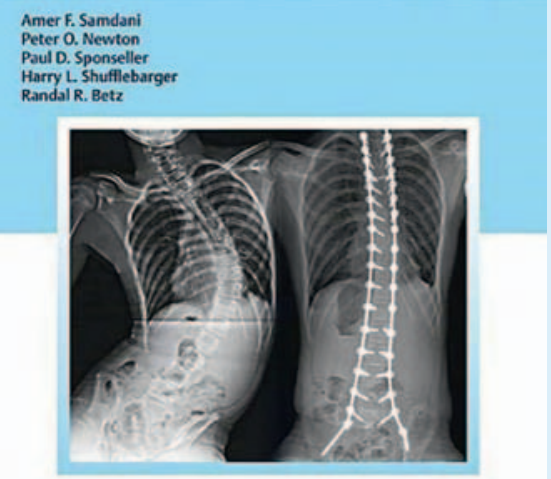

(4) Thieme

Amer F. Samdani, Peter O. Newton, Paul D. Sponseller, Harry L. Shufflebarger, Randal R. Betz, 206 Seiten, Thieme Medical Publishers, 1. Auflage, 2018, ISBN-13: 978-1626232600, 84,00€
Dieses englischsprachige Buch aus dem Hause Thieme befasst sich in 4 Kapiteln auf insgesamt 186 Seiten mit dem komplexen Gebiet der Skoliose-Chirurgie und spricht dementsprechend vor allem kinderchirurgisch tätige bzw. allgemein wirbelsäulenchirurgische Kollegen an.

Kapitel 1 befasst sich mit der allgemeinen präoperativen Beurteilung, konservativen Maßnahmen sowie den intraoperativen Gegebenheiten wie Anästhesie und Neuromonitoring. Außerdem werden operative Herausforderungen wie auch Komplikationen erörtert.

Im 2. Kapitel wird auf spezifische Erkrankungsentitäten eingegangen, wie Skoliose bei der Zerebralparese, spinale Deformitäten bei Myelomeningozelen, Muskeldystrophien, muskuläre Atrophien und andere seltene neuromuskuläre Erkrankungen. Auch spinale Deformitäten assoziiert mit neurodegenerativen Erkrankungen im Erwachsenenalter werden thematisiert.

Kapitel 3 beinhaltet die unterschiedlichen operativen Techniken und Zugangswege und im letzten 4. Kapitel wird auf das postoperative Management sowie Komplikationen eingegangen.

Jedes Unterkapitel ist übersichtlich von verschiedenen Autoren mit abschließendem Literaturverzeichnis aufgebaut, die einzelnen Themengebiete sind relativ knapp gehalten. Insgesamt finden sich 264 Illustrationen, vornehmlich Patientenbilder sowie prä- und postoperative Röntgenbilder, in diesem Buch, aber auch vereinzelte intraoperative Aufnahmen werden dargestellt.

Eine enge Zusammenarbeit besteht mit der Harms Study Group, einer weltweit organisierten Assoziation spinaler Chirurgen, um die Qualität in spinaler Kinder- und Erwachsenenchirurgie zu optimieren und nach bestem Wissensstand und neuesten Forschungsentwicklungen durchzuführen.

Gerade für operativ tätige Kollegen ist dieses Buch sicherlich ein hervorragendes Nachschlagewerk, um Fehlerquellen zu vermeiden und Therapiemöglichkeiten zu optimieren. Auch Radiologen mit Interesse an der Wirbelsäulenchirurgie sowie der pädiatrischen spinalen Chirurgie finden hier wissenswerte Informationen, um entsprechende radiologische Befunde den relevanten Aspekten anzupassen. Allgemein sind Radiologen allerdings sicherlich nicht Zielgruppe dieses Buches.

Lesenswert ist dieses Nachschlagewerk für alle, die sich mit jungen wie auch älteren Skoliose-Patienten befassen und einen Einblick in die aktuelle operative Chirurgie gewinnen möchten sowie aktuelle Literatur zum Outcome nach einer OP suchen.

Dr.med. Maya Woite

Ratingen 


\section{MRT-Einstelltechniken und -Protokolle}

Edition Radiopraxis

MRT Einstelltechniken

und Protokolle

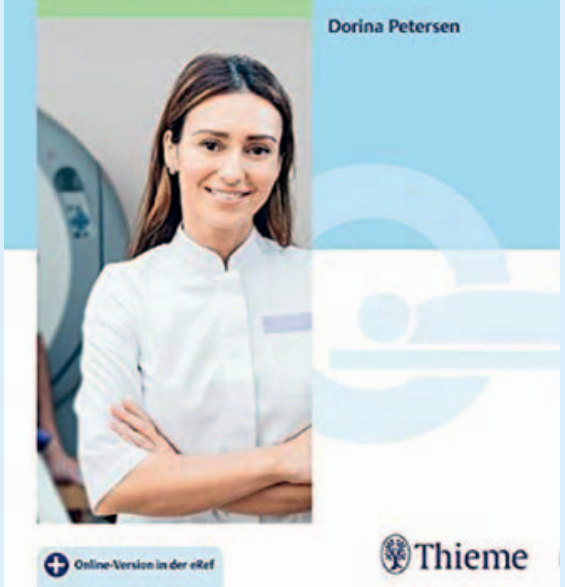

Edition Radiopraxis, Dorina Petersen, 380 Seiten, 664 Abbildungen, Thieme Verlag, 2020, Paperback, 1. Auflage 2020, ISBN: 978-3-13-242158-5, 49,99 Euro

Die MRT ist ein komplexes, anspruchsvolles bildgebendes Verfahren. Im Alltag wird von der MTRA oft erwartet, dass sie die Untersuchung eigenverantwortlich und selbstständig durchführt. Damit trägt eine gut ausgebildete und eingearbeitete MTRA sehr wesentlich zur Qualität von Untersuchung und Befund bei, sie trägt eine hohe Verantwortung.
Im ersten Teil des Buches werden von der Autorin auf 40 Seiten die wichtigsten Grundlagen wie MRT-Physik, Gerätetechnik, Kontrastmittel und MRT-Sicherheit auf gut verständliche Weise prägnant erklärt und beschrieben. Es wird auf Artefaktreduktion und Sequenzoptimierung eingegangen.

Der zweite Teil des Buches enthält Untersuchungsprotokolle/-empfehlungen für alle Körperregionen und Untersuchungsanforderungen. Dabei werden nicht nur die gängigen Fragestellungen wie Extremitäten, Gelenke, Wirbelsäule und Kopf erläutert, sondern auch seltene Untersuchungsregionen wie die Kiefergelenke mit Planung parasagittaler und parakoronarer Ebenen und das Sternum mit entsprechenden Bildbeispielen beschrieben.

Die Kapitel Untersuchungsprotokolle sind in logischer Weise unterteilt, was der täglichen Praxis entspricht:

- Vor der Untersuchung

Häufige Indikationen werden hier zusammengefasst und die Vorbereitung des Patienten mit Erläuterungen und Beispielfotos vorgestellt. Besonders hervorzuheben sind Erklärungen zu besonderen Situationen (zum Beispiel MRT des Kopfes bei Patienten, die nicht auf dem Rücken liegen können) mit alternativer Lagerung.

- Untersuchungsprotokoll und Planung In Listenform werden Beispielsequenzen in der üblichen Orientierung dargestellt. Diese sind so aufgelistet, dass sie wie eine Liste von Sequenzen auf dem Gerät aussehen, sodass ein direkter Vergleich leicht möglich ist. Direkt darunter befindet sich eine Tabelle mit den Sequenznamen der verschiedenen gängigen Hersteller GE, Siemens, Philips, Canon und Hitachi. In einer zweiten Tabelle wird der Zweck jeder Sequenz erklärt. Diese Informationen sind von hohem Wert und beantworten die wichtigen Fragen: Warum fährt man diese Sequenzen? Was wollen wir mit dieser Sequenz abbilden? Im Weiteren werden anhand von vielen Bildern die Planungsebenen visualisiert und im zugehörigen Text mit Tipps und Tricks beschrieben. Die Kontrast- und Auflösungsparameter für die Planung einer Sequenz sind am Ende eines jeden Kapitels in komprimierter und übersichtlicher Tabellenform aufgeführt.

Damit ist dieses Buch eines, das am Gerät schnell geöffnet werden kann und es einfach macht, den besten Weg zur Planung einer Untersuchung zu finden. Meinen MTRAs und angehenden RadiologInnen dient es als Leitfaden und Orientierung zur Durchführung von MRT-Untersuchungen in jeder Situation des Alltags. Ich kann dieses Buch nachdrücklich empfehlen, an jedem MRT-Arbeitsplatz parat zu haben. Es kann sowohl für Anfänger als auch für erfahrene Personen eine große Hilfe sein.

Dr. med. Julia Kalinka-Grafe Berlin 